\title{
Enhanced UV third-harmonic generation in microfibers by controlling nonlinear phase modulations
}

\author{
Xiujuan Jiang ${ }^{1, * \dagger}$, , Timothy Lee ${ }^{2,+}$, Mengmeng Chen ${ }^{3}$, Qi Sun ${ }^{2}$, Jing He $^{2}$, and Gilberto \\ BRAMBILLA $^{2}$ \\ ${ }^{1}$ School of Electromechanical Engineering, Guangdong University of Technology, Guangzhou, 510006, China \\ ${ }^{2}$ Optoelectronics Research Center, University of Southampton, Southampton, SO17 1BJ, United Kingdom \\ ${ }^{3}$ School of Electronics Engineering, Nanjing Xiaozhuang University, Nanjing 211171, China \\ ${ }^{*}$ Corresponding author: jiangxj@gdut.edu.cn
}

Compiled July 24, 2019

\begin{abstract}
The influence of nonlinear phase modulations on third harmonic conversion in silica microfibers is experimentally demonstrated. By utilizing such influence, enhanced narrow-bandwidth UV is generated at high signal-to-noise ratio $(33 \mathrm{~dB})$ and an average power of several hundred nanowatts. Detailed trends of third harmonic power against input pump power were characterized with peak pump power up to $2.5 \mathrm{~kW}$, and the results agree with predicted features, confirming that harmonic output could be optimized with adaptive control of phase mismatch. () 2019 Optical Society of America
\end{abstract}

OCIS codes: (190.0190) Nonlinear optics; (190.2620) Harmonic generation and mixing; (190.4370) Nonlinear optics, fibers.

\section{http://dx.doi.org/10.1364/ao.XX.XXXXXX}

Third harmonic $(\mathrm{TH})$ generation is a useful way of producing new laser wavelengths. Although fiber lasers are developing rapidly today with extensive and diverse applications, they still have to employ nonlinear crystals for frequency conversion. Compared with such hybrid solutions, an all-fiber system would be more robust and cost-effective. In fact, since TH was observed in an elliptical-core silica fiber [1], people have explored this phenomenon in different kinds of fibers, like microstructured fibers $[2,3]$, microfibers $[4,5]$ and highly doped fibers [6]. However, more work is needed to provide a deeper insight into the nonlinear process and to boost the conversion efficiency.

A difficult issue in achieving efficient $\mathrm{TH}$ with fibers is to correct the dynamic phase detuning caused by self-phase modulation (SPM) and cross-phase modulation (XPM). Without these effects, there would be a cubic evolution of $\mathrm{TH}$ power in the undepleted pump approximation, i.e., $P_{3} \propto P_{0}^{3}$ [4]. However, the phase matching is no longer satisfied when SPM/XPM effects become significant, resulting in harmonic power peaking and then oscillating which invalidates the aforementioned power relation. This occurs due to increased pump power, or longer interaction distance; the former has been noted experimentally [6] and the latter theoretically [7]. Our recent work gives a detailed analysis on the TH performance when both the pump power and the interaction distance are taken into account [8].
Intermodal phase-matching approaches require precise fiber diameters that are challenging to fabricate [9]. Fortunately, the ability to adjust pump parameters independent of fiber geometry provides potential solutions to this problem. For example, tuning the pump wavelength has already been proposed $[6,10]$, but this method is often restricted by narrow laser tuning range and amplifier gain spectrum limits.

Comparatively, an easier and more flexible control can be conducted on the input pump power. We have theoretically demonstrated that $\mathrm{TH}$ generation could be optimized by jointly controlling the microfiber parameters and input pump power to minimize the total phase mismatch, hence efficient TH could still be achieved with a microfiber with random roughness along its length [8]. In this letter, we report the experimental verification of such an idea. Silica microfibers with air cladding are designed and fabricated, and they are pumped with $2.3 \mathrm{~ns}, 200 \mathrm{kHz}$ laser pulses at $1091 \mathrm{~nm}$. Enhanced ultraviolet (UV) third harmonic is generated at average power up to several hundred nanowatts, and its detailed trend against input pump power agrees with the prediction.

The TH generation process in a microfiber is modeled with the coupled-mode equations, the lossless version of which can be accessed in [4], and the version including loss in [8]. The basic phase matching diameters can be found by solving the rigorous modal eigenvalue equations for a step index profile [11]. Figure 1(a) shows dependence of effective refractive index $n^{\text {eff }}$ of several modes on the microfiber diameter. The propagation constant of the mode is defined as $\beta=(\omega / c) n$ eff, and $\delta \beta=\beta_{3}-3 \beta_{1}$ is the mismatch between the pump and its TH. Now we choose to phase match the modes $\operatorname{HE}_{11}\left(\omega_{1}\right)$ and $\operatorname{HE}_{12}\left(3 \omega_{1}\right)$, and the basic diameter should be $d_{0}=521.3 \mathrm{~nm}$, yielding $\delta \beta\left(d_{0}\right)=0$.

Including the effects of SPM/XPM, the total propagation constant mismatch is $\delta \beta^{\prime}=\delta \beta+\delta \beta_{N L}$, and the nonlinear detuning can be approximated as $\delta \beta_{N L} \approx 3 k_{1} n^{(2)}\left(2 J_{2}-J_{1}\right) P_{0}$. Here $P_{0}$ is the input pump power, $k_{1}=2 \pi / \lambda_{1}$ is the pump propagation constant in vacuum, and $n^{(2)}$ is the nonlinear refractive index coefficient $\left(n^{(2)}=2.7 \times 10^{-20} \mathrm{~m}^{2} / \mathrm{W}\right.$ for silica). $J_{1}$ and $J_{2}$ are nonlinear overlap integrals governing SPM of the pump and XPM between the pump and the harmonic, respectively. Calculations of the nonlinear overlap integrals are given in [4], and further discussion about $\delta \beta_{N L}$ is presented in [8]. 

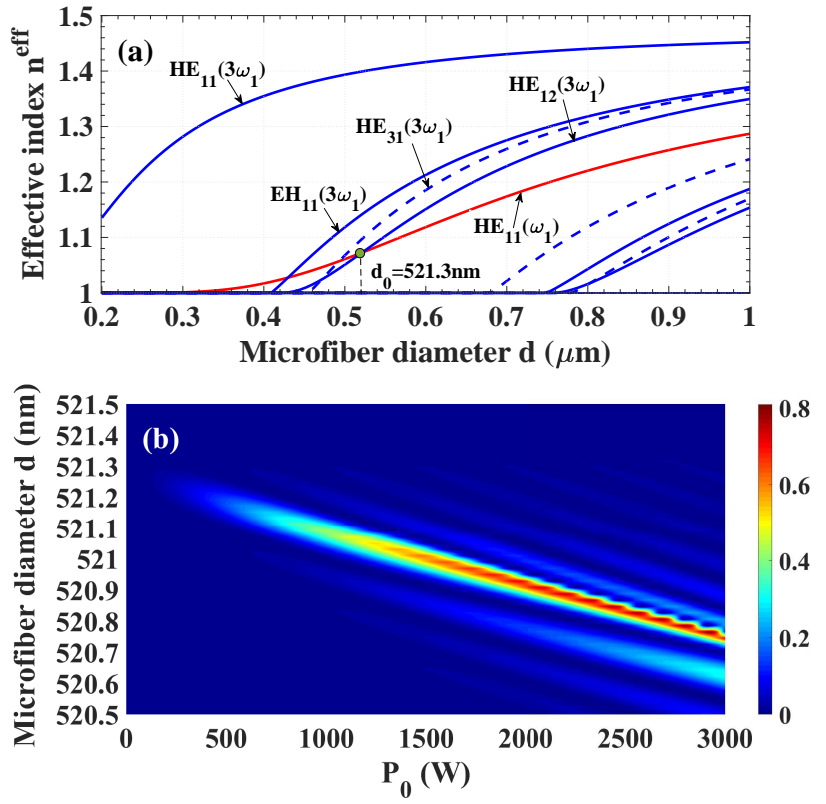

Fig. 1. (a) Dependence of effective refractive index on silica microfiber diameter $d$ for the fundamental pump mode $\mathrm{HE}_{11}\left(\omega_{1}\right)$ (red) and for the third harmonic hybrid $\mathrm{HE}_{v m}$ and $\mathrm{EH}_{v m}$ modes with azimuthal order $v=1$ (solid blue) and $v=3$ (dashed blue). The pump wavelength is $1091 \mathrm{~nm}$. (b) Output conversion efficiency of an 8-mm-long uniform and lossless microfiber against $d$ and the input pump power $P_{0}$.

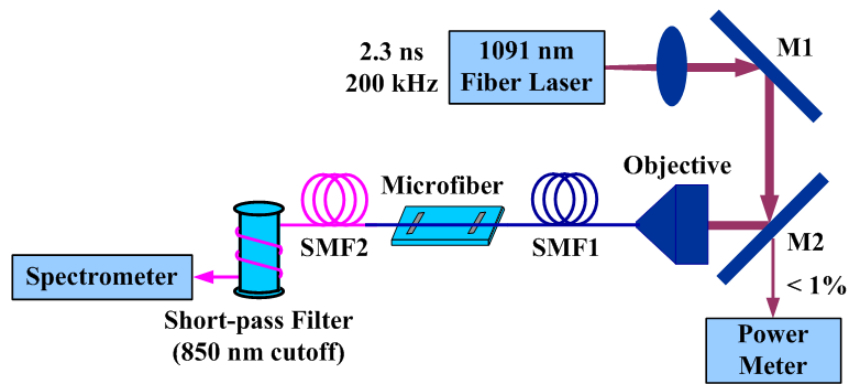

Fig. 2. Schematic of the experiment setup.

A modification of diameter can be made to offset the nonlinear detuning so that $\delta \beta^{\prime} \approx 0$. In previous work [4, 8], an initial value of $P_{0}$ was set to estimate $\delta \beta_{N L}$ when deciding this modification. However, we note here that a given change in $P_{0}$ is equivalent to a variation of the diameter in terms of effect on the phase matching, and thus the TH could actually be optimized with their proper combination. The output TH conversion efficiency of a uniform and lossless microfiber against these two parameters is shown in Fig. 1(b). It can be seen that, at a fixed pump power, the conversion is efficient over a very narrow diameter range which demands high tolerance of about $0.1 \mathrm{~nm}$, but an adjustment of $\sim 3000 \mathrm{~W}$ on $P_{0}$ could improve it to about $0.5 \mathrm{~nm}$. The former tolerance is impossible to achieve using silica glass as it is comparable to molecular dimensions, but the latter would fall in the range over which the efficiency exceeds 1\% [12]. Furthermore, while the microfiber diameters are unlikely to be made with adequate repeatability even when the tolerance is reduced to $\sim 0.5 \mathrm{~nm}$, an adaptive adjustment on $P_{0}$ can be made flexibly since it is independent of fiber structure.
Therefore, Fig. 1(b) indicates a favorable relation that relaxes the strict requirements in the microfiber design and fabrication.

Because the TH oscillates along the propagation distance due to SPM/XPM, the output of a specific microfiber with certain diameter and length will vary with $P_{0}$. Figure $1(\mathrm{~b})$ shows a high efficiency of about $80 \%$ with an ideally uniform microfiber. When random diameter fluctuations are taken into account, the output would be reduced by one or two orders of magnitude, but the trend would be similar [8].
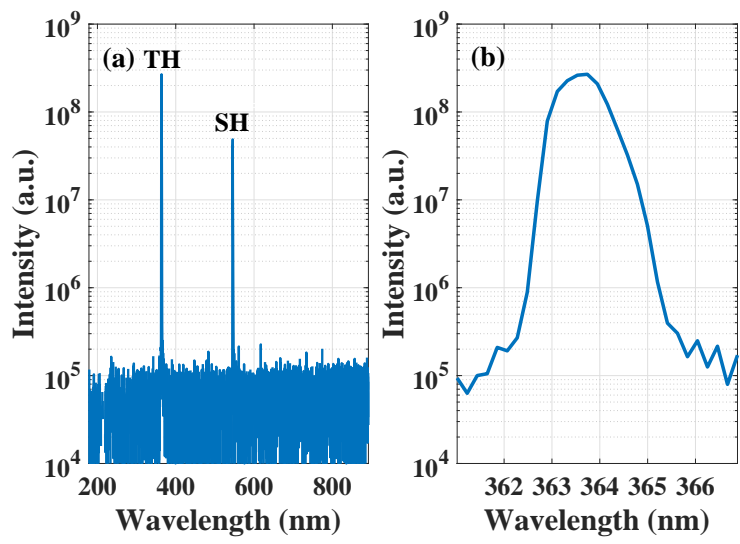

Fig. 3. (a) Third and second harmonic signals of the $1091 \mathrm{~nm}$ pump detected with the spectrometer; (b) details of the third harmonic spectrum. Integration time $\tau=0.1 \mathrm{~ms}$. The pump power is $2600 \mathrm{~W}$ at peak and $1.2 \mathrm{~W}$ at average. The microfiber is $521.0 \mathrm{~nm}$ in diameter and $6 \mathrm{~mm}$ in length (Taper A).

Based on the above simulations and possible uncertainty in tapering, two silica microfiber samples were made from Corning SMF-28: Taper A with diameter $d=521.0 \mathrm{~nm}$ and length $L=6 \mathrm{~mm}$; Taper B with $d=520.2 \mathrm{~nm}$ and $L=8 \mathrm{~mm}$. Each was removed from the tapering rig after it was fabricated (the rig was described with detail in [10]). The microfiber waist was suspended freely in the air, with the two untapered ends fastened on the raised parts of a metal strip, which was then fixed in a plastic box to minimize contamination of the taper.

The experiment setup is shown in Fig. 2. The $1091 \mathrm{~nm}$ fiber laser of $0.16 \mathrm{~nm}$ FWHM bandwidth with an adjustable power is used as the pump source [13]. About $50 \%$ of its power is coupled through the objective into SMF1 (Corning SMF-28), which is then spliced to the input end of the microfiber. The microfiber output is spliced to SMF2, a pure silica core single mode fiber (Sumitomo, Z Fiber ITU-T G.654.C, about two meters) designed to reduce UV absorption. A segment of SMF2 is made into a short-pass filter by wrapping it around a rod (the bend loss attenuates the pump while transmitting the harmonic). The harmonic signals are detected with the spectrometer (OceanOptics, USB4000-UV-VIS, $1 \mathrm{~nm}$ resolution). A small portion of the pump power $(<1 \%)$ is measured with the detector (Thorlabs S130VC) placed behind dichroic mirror M2 to monitor pump stability. Fabrication and testing of the microfibers are carried out under ordinary lab conditions at $23{ }^{\circ} \mathrm{C}$ and $20 \%$ relative humidity.

Figure 3 shows the clear TH signal when the laser pumps Taper A. The spectrum is fairly clean peaking at $363.7 \mathrm{~nm}$ and the FWHM bandwidth is $\sim 1 \mathrm{~nm}$, probably resolution-limited by the spectrometer. At $1.2 \mathrm{~W}$ average ( $2600 \mathrm{~W}$ peak) pump power, the TH power is $\sim 508 \mathrm{nW}$. The output-input conversion efficiency, defined as the ratio of average output $\mathrm{TH}$ power to 

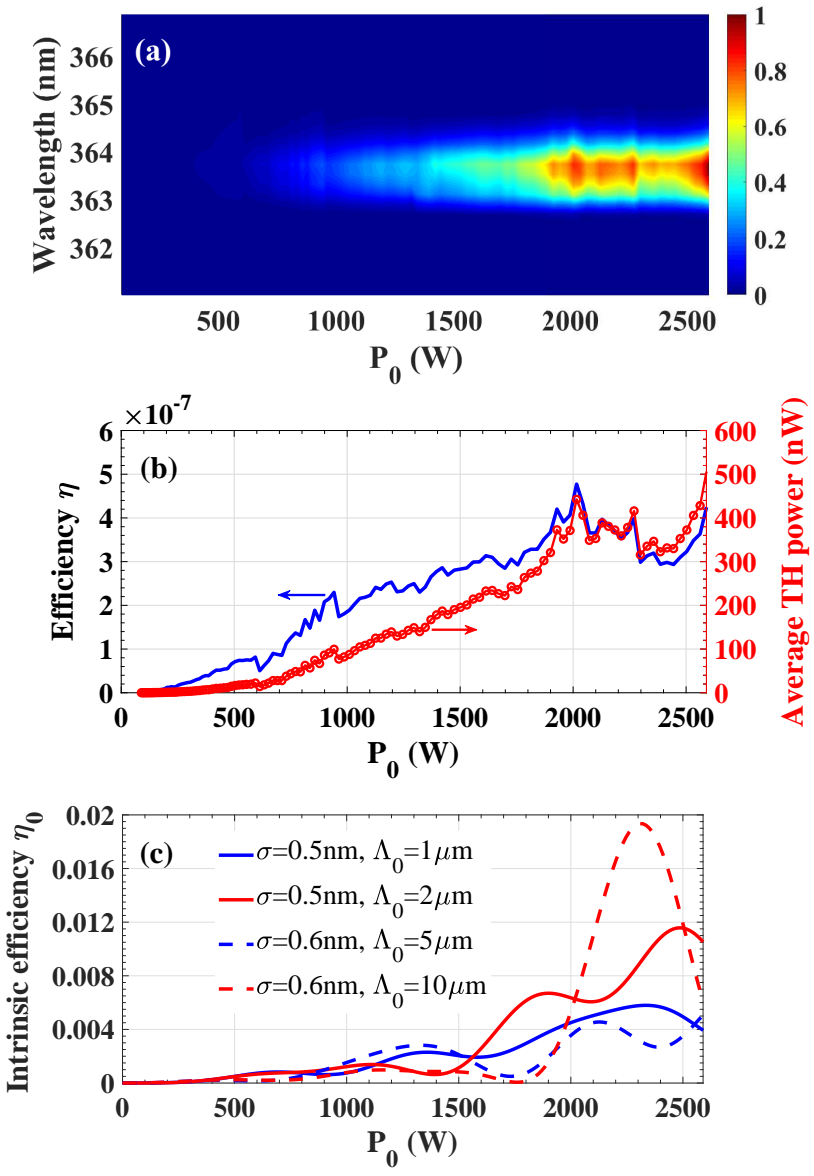

Fig. 4. $\mathrm{TH}$ against pump peak power $P_{0}$ for Taper $\mathrm{A}(d=$ $521.0 \mathrm{~nm}, L=6 \mathrm{~mm}$ ): (a) Output spectrum (maximum intensity normalized to unity). (b) Average output TH power integrated over $361<\lambda<367 \mathrm{~nm}$ (red) and corresponding efficiency (blue). (c) Simulated intrinsic efficiency for different microfiber surface roughness (microfiber losses included).

average input pump power of the whole fiber system, is thus $\eta \approx 4 \times 10^{-7}$. The intrinsic efficiency of TH generation only over the microfiber, denoted as $\eta_{0}$ here, should be higher than this overall 'net' efficiency when losses in other parts of the setup are considered (more discussion will be made on this issue later). The shown signal-to-noise ratio (SNR) for $\mathrm{TH}$ is about $2000: 1$ or $33 \mathrm{~dB}$, but this value is based on the measurements with short integration time because the spectrometer provides limited dynamic range for a single acquisition, and the true SNR may be much higher. As seen in Fig. 3(a), the second harmonic $(\mathrm{SH})$ at $545.6 \mathrm{~nm}$ is also detected with a power one fifth of that of the $\mathrm{TH}$, and it is believed to arise due to bulk multipole nonlinearities and surface dipole [14].

The peak power of the pump is adjusted in steps of around $25 \mathrm{~W}$, and the resulting harmonic spectra are recorded. Figure 4(a) shows the output TH spectrum against $P_{0}$ for Taper A. The spectrum profile is basically uniform throughout, with the center remaining at $363.7 \mathrm{~nm}$ and the bandwidth about $1 \mathrm{~nm}$. The TH power integrated over the range $361-367 \mathrm{~nm}$ is presented in Fig. 4 (b). When $P_{0}<2000 \mathrm{~W}$, the TH power goes up roughly monotonically with increasing pump power; beyond that, obvious oscillation appears but the TH performance could be optimized at certain pump powers. The output-input TH efficiency $\eta$ is also calculated. The simulated intrinsic efficiency in Fig. 4(c) will be discussed later. The counterparts for Taper B are given in Fig. 5, and they exhibit more oscillations with increasing pump power as Taper B is longer.

These results are in agreement with theoretical predictions [8]. At low pump power, the relation $P_{3} \propto P_{0}^{3}$ could be valid; when the pump power is high and the SPM/XPM effects cannot be ignored, coupled with diameter fluctuation of the real microfiber, the TH behavior is complicated and the output oscillates with the input pump power. Nevertheless, when the total phase detuning, consisting of the linear part dominated by microfiber diameter and the nonlinear part caused by SPM/XPM, is minimized, improved TH performance is still achieved.
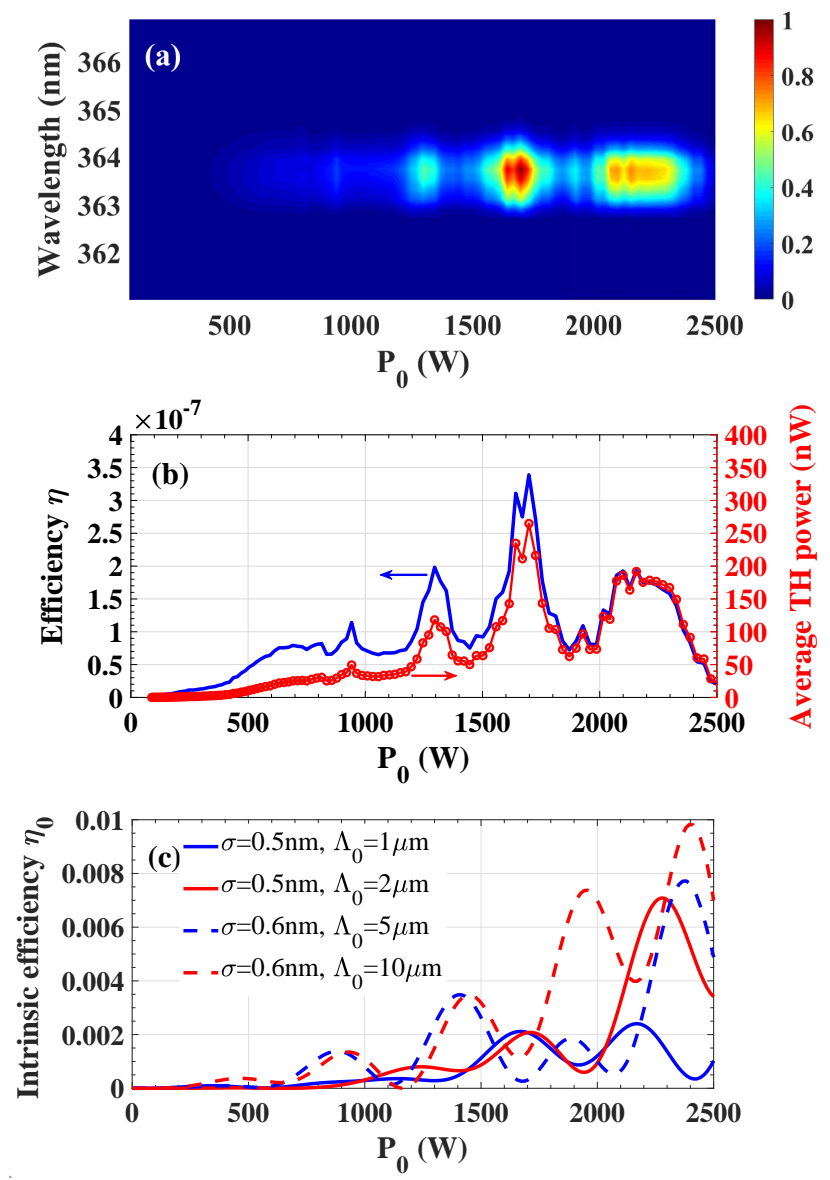

Fig. 5. TH against peak input pump power $P_{0}$ for Taper B $(d=520.2 \mathrm{~nm}, L=8 \mathrm{~mm})$. Other descriptions are as in Fig. 4 .

With a waist diameter about $520 \mathrm{~nm}$, the silica microfibers are fragile and damage usually occurs if they are subjected to weak mechanical shock like airflow or accidental contact. However, they are highly robust against powerful laser pulses once packaged. For example, Taper B worked continuously for more than two hours without damage, withstanding tests at $550 \mathrm{~W}$ peak pump power for 30 minutes, $1200 \mathrm{~W}$ for another $30 \mathrm{~min}$ utes, and then $2000 \mathrm{~W}$ for over 60 minutes. The lifetime of a microfiber depends on the quality of fabrication and packaging. These experiments were undertaken in ordinary labs exposed to flowing air with dust throughout the whole process; if the microfibers were to be fabricated in a cleanroom and packaged properly in an air-tight box, their lifetime would be extended 
and performance could be better.

In this work, narrow-bandwidth $\mathrm{TH}$ is generated with hundred-nanowatts average power and SNR over $33 \mathrm{~dB}$, which, to our knowledge, represents a notable improvement for UV generation in such microfibers. Unlike the complicated spectra obtained in many experiments $[1-3,10]$, the spectrum here is very simple and clean, and it may probably benefit from the short microfiber length which prevents other competing nonlinear processes, e.g. stimulated Raman scattering and four-wave mixing. This spectrum is actually similar to that reported in [5], but the output is enhanced by two orders of magnitude compared to its maximum $\mathrm{TH}$ power of just $7 \mathrm{nW}$. In our previous work [15], deep UV was generated based on TH in a microfiber too, yielding an average power of $0.15 \mathrm{nW}$ and SNR lower than ten, and now significant improvement has been made here.

The TH efficiency of $\sim 10^{-7}$ is comparable to other experiments $[3,5,10]$, but it is still rather low. As diameter deviation would reduce the efficiency, we performed simulations to analyse the output of Taper A and Taper B, see Figs. 4(c) and 5(c). Because surface parameters of the tapers could not be known exactly, several different degrees of roughness were tried. Both the roughness amplitude and length are assumed to obey Gaussian distributions, in which $\sigma$ is width of the amplitude distribution and $\Lambda_{0}$ is the median roughness length (more details in [8]). A fiber of larger $\sigma$ and shorter $\Lambda_{0}$ is rougher. The existing work suggests a roughness $\sim 0.3 \mathrm{~nm}$ [9]; when we use conservative values $\sigma=0.5 \mathrm{~nm}$ and $0.6 \mathrm{~nm}$, intrinsic TH efficiency $\eta_{0}$ could still reach $10^{-3}-10^{-2}$. These simulations give estimates on the order of magnitude for possible efficiency; meanwhile, the curve trends are close to the experimental results, although none of them matches the latter completely as random roughness of the tapers cannot be copied, confirming that theoretical analyses in this letter and in [8] are reliable.

The silica microfibers were pumped at $1550 \mathrm{~nm}$ in [8], with a power loss of $2 \mathrm{~dB} / \mathrm{m}$ for the pump and $20 \mathrm{~dB} / \mathrm{m}$ for its $\mathrm{TH}$; now the $1091 \mathrm{~nm}$ pump would experience greater material absorption but less scattering loss as it would be confined more tightly, so we also use the same estimates for loss in simulating Figs. 4(c) and 5(c). The TH output is lower when simulating with greater losses, but the difference is not large as the microfibers are short, e.g., if using $4 \mathrm{~dB} / \mathrm{m}$ for the pump and $60 \mathrm{~dB} / \mathrm{m}$ for the $\mathrm{TH}$ in Taper B, the peaks of the curve with $\sigma=0.6 \mathrm{~nm}$ and $\Lambda_{0}=10 \mu \mathrm{m}$ are reduced by no more than $30 \%$.

Therefore, very low conversion efficiency may not result from surface roughness and losses in the microfiber, and the measured efficiency might actually be reduced by $\sim 10^{4}$ due to losses in the setup. The major losses may include: (i) About $30 \mathrm{~dB}$ UV absorption in the one-meter untapered SMF-28 fiber on the output end of the microfiber [16, 17]; (ii) About $7 \mathrm{~dB}$ UV absorption in the two-meter $\mathrm{Z}$ fiber [15]; (iii) High-order-mode UV attenuation due to bend loss in the delivery fibers, around $3 \mathrm{~dB}$.

In fact, because the pump and third harmonic wavelengths are far apart and they propagate in different modes, to deliver both at low loss in the same fiber would be a huge challenge, as pointed out in [4]. This might be a key reason why the detected TH generation efficiency is very low in experiments so far. It is worth studying this important issue further to improve the delivery/collection method, and the results will be reported in a separate paper.

One difficulty in this work is to fabricate a microfiber with the exact diameter. If the waist is narrower than the critical diameter, phase matching will occur in the transition region, but the effective interaction distance (over which the diameter is within $\sim 0.5 \mathrm{~nm}$ of the critical value) would be rather short due to the transition slope, in the range $0.1-1 \mathrm{~mm}$, resulting in low efficiency. Alternatively, TH would be more efficient by phase matching over the waist, assuming the diameter deviation is small enough to be compensated by pump power adjustment. Given the fabrication uncertainty of the tapering rig and by altering the parameters carefully, one microfiber of suitable diameter could be made out of several trials. Note that while we set the design parameters for a microfiber on the rig, it is hard to characterize its precise diameter and uniformity after the fabrication - confirmation by SEM imaging was only conducted after testing as it damages the taper. This is also problematic for related wave mixing processes, and we are now working on new schemes which further improve diameter error tolerance.

To conclude, the influence of nonlinear phase modulations on microfiber-based TH generation is experimentally demonstrated. The harmonic performance is optimized by adjusting the input pump power to minimize the total phase detuning. Enhanced narrow-bandwidth $(\sim 1 \mathrm{~nm}) \mathrm{UV}$ is achieved at high SNR of 33 $\mathrm{dB}$ and average power over $500 \mathrm{nW}$. The silica microfibers about $520 \mathrm{~nm}$ in diameter are proven to be robust when pumped with ns pulses at a peak power over $2000 \mathrm{~W}$. This work provides more insight into the third harmonic conversion process in fibers and brings it closer to practical exploitation.

\section{FUNDING}

China Scholarship Council (201308440325); Guangdong Provincial Natural Science Foundation (2018A030313315); Engineering and Physical Sciences Research Council (EP/L01243X/1); Royal Society (IE131732).

\section{ACKNOWLEDGMENTS}

The authors thank Dr. Rand Ismaeel for instrument assistance.

\section{REFERENCES}

1. J. M. Gabriagues, Opt. Lett. 8, 183 (1983).

2. A. Efimov, A. J. Taylor, F. G. Omenetto, J. C. Knight, W. J. Wadsworth, and P. St. J. Russell, Opt. Express 11, 2567 (2003).

3. S. C. Warren-Smith, K. Schaarschmidt, M. Chemnitz, E. P. Schartner, H. Schneidewind, H. Ebendorff-Heidepriem, and M. A. Schmidt, Opt. Lett. 44, 626 (2019).

4. V. Grubsky and A. Savchenko, Opt. Express 13, 6798 (2005).

5. V. Grubsky and J. Feinberg, Opt. Commun. 274, 447 (2007).

6. K. Bencheikh, S. Richard, G. Mélin, G. Krabshuis, F. Gooijer, and J. A. Levenson, Opt. Lett. 37, 289 (2012).

7. X. Jiang, T. Lee, J. He, M. I. M. A. Khudus, and G. Brambilla, Opt. Express 25, 22626 (2017).

8. X. Jiang, D. Zhang, T. Lee, and G. Brambilla, Opt. Lett. 43, 2728 (2018).

9. M. I. M. A. Khudus, T. Lee, P. Horak, and G. Brambilla, Opt. Lett. 40, 1318 (2015).

10. T. Lee, Y. Jung, C. A. Codemard, M. Ding, N. G. R. Broderick, and G. Brambilla, Opt. Express 20, 8503 (2012).

11. A. Snyder and J. Love, Optical Waveguide Theory, 1st ed. (Springer, 1983).

12. T. Lee, Thesis for the degree of Doctor of Philosophy (University of Southampton, 2013).

13. J. He, D. Lin, L. Xu, M. Beresna, M. N. Zervas, S. Alam, and G. Brambilla, Opt. Express 26, 6554 (2018).

14. J. Lægsgaard, J. Opt. Soc. Am. B 27, 1317 (2010).

15. Y. Wang, T. Lee, F. De Lucia, M. I. M. Abdul Khudus, P. J. A. Sazio, M. Beresna, and G. Brambilla, Opt. Lett. 42, 4671 (2017).

16. R. Kitamura, L. Pilon, and M. Jonasz, Appl. Opt. 46, 8118 (2007).

17. U. Wiedemann, K. Karapetyan, C. Dan, D. Pritzkau, W. Alt, S. Irsen, and D. Meschede, Opt. Express 18, 7693 (2010). 


\section{REFERENCES}

1. J. M. Gabriagues, "Third-harmonic and three-wave sum-frequency light generation in an elliptical-core optical fiber," Opt. Lett. 8(3), 183-185 (1983).

2. A. Efimov, A. J. Taylor, F. G. Omenetto, J. C. Knight, W. J. Wadsworth, and P. St. J. Russell, "Phase-matched third harmonic generation in microstructured fibers," Opt. Express 11(20), 2567-2576 (2003).

3. S. C. Warren-Smith, K. Schaarschmidt, M. Chemnitz, E. P. Schartner, H. Schneidewind, H. Ebendorff-Heidepriem, and M. A. Schmidt, "Tunable multi-wavelength third-harmonic generation using exposed-core microstructured optical fiber," Opt. Lett. 44(3), 626-629 (2019).

4. V. Grubsky and A. Savchenko, "Glass micro-fibers for efficient third harmonic generation," Opt. Express 13(18), 6798-6806 (2005).

5. V. Grubsky and J. Feinberg, "Phase-matched third-harmonic UV generation using low-order modes in a glass micro-fiber," Opt. Commun. 274(2), 447-450 (2007).

6. K. Bencheikh, S. Richard, G. Mélin, G. Krabshuis, F. Gooijer, and J. A. Levenson, "Phase-matched third-harmonic generation in highly germanium-doped fiber," Opt. Lett. 37(3), 289-291 (2012).

7. X. Jiang, T. Lee, J. He, M. I. M. A. Khudus, and G. Brambilla, "Fundamental-mode third harmonic generation in microfibers by pulseinduced quasi-phase matching," Opt. Express 25(19), 22626-22639 (2017).

8. X. Jiang, D. Zhang, T. Lee, and G. Brambilla, "Optimized microfiberbased third-harmonic generation with adaptive control of phase mismatch," Opt. Lett. 43(12), 2728-2731 (2018).

9. M. I. M. A. Khudus, T. Lee, P. Horak, and G. Brambilla, "Effect of intrinsic surface roughness on the efficiency of intermodal phase matching in silica optical nanofibers," Opt. Lett. 40(7), 1318-1321 (2015).

10. T. Lee, Y. Jung, C. A. Codemard, M. Ding, N. G. R. Broderick, and G. Brambilla, "Broadband third harmonic generation in tapered silica fibres," Opt. Express 20(8), 8503-8511 (2012).

11. A. Snyder and J. Love, Optical Waveguide Theory, 1st ed. (Springer, 1983).

12. T. Lee, "Nonlinear properties of optical microfibres," Thesis for the degree of Doctor of Philosophy (University of Southampton, 2013).

13. J. He, D. Lin, L. Xu, M. Beresna, M. N. Zervas, S. Alam, and G. Brambilla, " $5.6 \mathrm{~kW}$ peak power, nanosecond pulses at $274 \mathrm{~nm}$ from a frequency quadrupled Yb-doped fiber MOPA," Opt. Express 26(6), 6554-6559 (2018).

14. J. Lægsgaard, "Theory of surface second-harmonic generation in silica nanowires," J. Opt. Soc. Am. B 27(7), 1317-1324 (2010).

15. Y. Wang, T. Lee, F. De Lucia, M. I. M. Abdul Khudus, P. J. A. Sazio, M. Beresna, and G. Brambilla, "All-fiber sixth-harmonic generation of deep UV," Opt. Lett. 42(22), 4671-4674 (2017).

16. R. Kitamura, L. Pilon, and M. Jonasz, "Optical constants of silica glass from extreme ultraviolet to far infrared at near room temperature," Appl. Opt. 46(33), 8118-8133 (2007).

17. U. Wiedemann, K. Karapetyan, C. Dan, D. Pritzkau, W. Alt, S. Irsen, and D. Meschede, "Measurement of submicrometre diameters of tapered optical fibres using harmonic generation," Opt. Express 18(8), 76937704 (2010). 